\title{
LOS FUNDAMENTOS RELIGIOSOS \\ DE LA BILDUNG COMO EPÍTOME \\ DE LA EDUCACIÓN ALEMANA Y SU FUNDAMENTAL RECELO ANTIOCCIDENTAL
}

\section{The religious foundations of Bildung as an epitome of German education and its fundamental anti-western recipe}

\author{
Daniel TRÖHLER \\ Universidad de Luxemburgo \\ Correo-e: daniel.troehler@uni.lu
}

Recepción: 8 de agosto de 20I5. Envío a informantes: Is de agosto de 2015.

Fecha de aceptación definitiva: II de octubre de 20I5

Resumen: Este artículo examina cómo durante la República de Weimar (1919I933), el pensamiento luterano permitió la incorporación de la educación como disciplina universitaria, organizada intelectualmente en torno a la noción de Bildung excluyendo aspectos prácticos. Con el fin de proporcionar un recorrido histórico este trabajo aborda el período entre 1890 y 1925 . En primer lugar, el artículo examina cómo, hacia el final del siglo xix, el crecimiento económico, territorial y militar de Alemania no fue favorecido por su intelectualidad, la cual sustentó una filosofía dualista. A continuación, el documento analiza el trasfondo cultural e institucional de esta filosofía en la que la teoría de la Bildung emergió y se desarrolló. En tercer lugar, el artículo reconstruye el modo en el que las ciencias de la educación,

\footnotetext{
Este artículo continúa con argumentos ya esgrimidos en: TRöHLER, D.: «The German idea of Bildung and the Anti-Western Ideology», en Siljander, P.; Kivelä, A. y Sutinen, A. (eds.): Theories of Bildung and Growth. Connections and Controversies between Continental European Educational Thinking and American Pragmatism, Rotterdam, Sense Publishers, 20I2, pp. I49-164.

Me gustaría agradecerle a Miguel A. Pereyra su inestimable ayuda en la organización de la traducción española.
} 
LOS FUNDAMENTOS RELIGIOSOS DE LA BILDUNG COMO EPÍTOME DE LA EDUCACIÓN

fundamentadas en la ideología de Bildung, se convirtieron en una disciplina académica durante la Gran Guerra. También se analiza cómo Persönlichkeit y Bildung se convirtieron en los reclamos dominantes en la batalla contra Occidente y las ciencias empíricas. Finalmente, el documento examina cómo los defensores de Bildung trataron de mantener su viabilidad.

Palabras clave: Bildung; ciencias de la educación; República de Weimar; Personlichkeit.

AвSTRACT: This article examines how during the Weimar Republic (19191933), Lutheran thinking enabled the incorporation of education as an university discipline, intellectually organized around the notion of Bildung excluding practical aspects. In order to provide a background the article deals with the period between I890 and 1925. First, the article examined how toward the end of the nineteenth century, Germany's economic, territorial, and military growth, was not favoured by its intelligentsia which held a dualistic philosophy. Secondly, the paper discusses the cultural and institutional background of this philosophy in which the theory of Bildung arose and grew. Thirdly, the paper reconstructs how educational sciences, based on the ideology of Bildung became an academic discipline, during the Great War and explains how Persönlichkeit and Bildung became dominant battle cries against the West and the empirical sciences. Finally, the paper examines how advocates of Bildung tried to keep its viability.

KEY WORDs: Bildung; educational sciences; Weimar Republic; Personlichkeit.

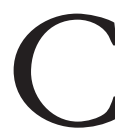

UANDO EN 2OOI SE PUBLICARON los primeros resultados del informe PISA, Alemania quedó consternada². Nadie habría pensado antes que Alemania no aparecería entre los diez primeros en resultados educativos, ya que -según estaba convencido todo el mundo- Alemania siempre había sido el país de la educación y la Bildung. Los debates públicos alumbraron la búsqueda de causas y culpables, y los políticos se vieron obligados a encontrar soluciones. En 2002 la Universidad de Heidelberg organizó una serie de lecturas públicas dedicadas al tema: «¿Somos todavía un pueblo de poetas y pensadores?». El subtítulo informaba a los miembros de la universidad de que en el semestre de invierno 2002/2003, el Studium generale de la universidad se centraría en cuestiones educativas (Bildungsfragen). Diez conferenciantes fueron invitados a participar, incluso un extranjero, tal y como anunciaba orgullosamente el anuncio, entre ellos filósofos, historiadores, políticos, escritores -y ninguno de ellos relacionado con las Ciencias de la Educación-. Educación y Bildung eran asunto de identidad nacional y no debían ser dejados a los educadores: «Aunque la mala posición en el informe PISA importa en el ámbito de la educación y escolarización», sigue la introducción

Los siguientes párrafos están más desarrollados en: TröHLeR, Daniel: «Conceptos, culturas y comparaciones: PISA y el doble descontento alemán», Profesorado, I7, 2 (2013a), pp. I27-I39. Disponible en: http://www.ugr.es/ recfpro/rev172ART8.pdf. 
a esta serie de conferencias, «las dudas internas van más allá de eso. Una nación entera se pregunta: ¿Somos aún el pueblo de los poetas y pensadores?»³.

De acuerdo con otras muchas reacciones de los intelectuales alemanes, la serie de conferencias de Heidelberg no identificó problemas particulares en el currículum o en el sistema educativo formal como causa de los pobres resultados de PISA, sino que se centró en el equivocado énfasis conceptual de PISA respecto a las habilidades y competencias. Wolff-Metternich ${ }^{4}$, filósofo, recordaba al público qué es Bildung -o mejor, qué no es Bildung: «Bildung... no es aprendizaje cifrable y fijable- tampoco teórica o prácticamente», afirmaba, no utilitario y no pragmático 5 , por tanto, principalmente sin propósito ${ }^{6}$. Frühwald 7 , profesor de estudios literarios, hizo una comparación con la teoría humboldtiana de Bildung e identificó las asunciones básicas de PISA, usando como metáfora médica el «cáncer» de la «ideología-válida-monetariamente» ${ }^{8}-y$ lo escribió en inglés-. Tal y como Hermann', historiador de Educación, afirmó algunos años más tarde, la Bildung no es medible y es, al mismo tiempo, un proceso y su resultado; no es aprendizaje ni competencia sino una transformación interna del alma con el resultado de una Persönlichkeit [personalidad] ${ }^{\mathrm{I}}$. La Persönlichkeit cono resultado de la Bildung es la persona autosuficiente, madura y equilibrada, mientras que PISA y su programación intentan incapacitar personas, con la pretensión de entrenarlas para ser obedientes homo oeconomicus ${ }^{\text {II }}$. La Bildung es el deseado objetivo de uno de los dos polos en una visión del mundo radicalmente dualista que distingue agudamente un mundo interior de un mundo exterior. Es la-digamos por el momento «cultural»- condición previa de una visión del mundo dualista que permite a la idea de Bildung y a la idea de Persönlichkeit convertirse en nociones polémicas contra, no tanto los resultados de PISA, como sus mismas intenciones y ajustes.

Como las referencias a Humboldt y a los poetas alemanes indican, los orígenes de la Bildung y la Persönlichkeit se sitúan alrededor de i8oo. En este punto es

3 Unispiegel der Universität Heidelberg: «Noch Volk der Dichter und Denker? Studium Generale beschäftigt sich im Wintersemester mit Bildungsfragen», 2002, I. Disponible en: http:// www.uni-heidelberg.de/presse/unispiegel/uso2-4/generale.html.

4 Wolff-Metternich, B.-S. von: «Was heißt heute: sich im Denken orientieren?», en Sind wir noch ein Volk der Dichter und Denker? Sammelband der Vorträge des Studium Generale der Universität Heidelberg im Wintersemester 2002/2003, Heidelberg, Universitätsverlag Winter, 2004, pp. 65-76, p. 68.

Ibidem, p. 69.

6 Ibidem, p. 7I.

7 FrÜHWALD, W: «Kulturenstreit oder Von der Rolle der Sprache in der Wissenschaft», en Tröger, J. y Must, H. (eds.): Sind wir noch ein Volk der Dichter und Denker?, Heidelberg, Universitätsverlag Winter, 2004, pp. 35-45.

8 Ibidem, p. 42.

9 Herrmann, Ulrich: «Bildung, Kompetenz - oder was? Einige notwendige Begriffsklärungen», Engagement. Zeitschrift für Erziehung und Schule, 3 (2007), pp. 171-178.

Io Ibidem, p. 172.

" Krautz, Jochen: «Die Vereinnahmung der Person: Zu Auswirkungen und Hintergründen des Kompetenz-Konzepts», Engagement. Zeitschrift für Erziehung und Schule, 3 (2007), pp. 2II-225, p. 216. 
importante señalar que el dicho de «los poetas y pensadores alemanes» ha sido polémico desde sus inicios, ya que fue usado en Francia por la gran burguesa Madame de Staël en su libro De l'Allemagne (De Staël, i8ıo), en el cual ella compara la pretendidamente profunda, cultural e intelectualmente superior Alemania con la Francia solo militarmente superior bajo las tropas de Napoleón (a las cuales ella odiaba por su igualitarismo). Esta característica polémica -y de alguna forma desafiante- permanecía como un rasgo inherente de la teoría alemana de la Bil$d u n g^{12}$, lo que puede ser descubierto no sólo en los debates de PISA posteriores a 2000 sino también -y quizá sobre todo- cien años, antes y durante la Gran Guerra (1914-1918) y el subsiguiente experimento que introducía la democracia en Alemania en la época de la República de Weimar (1919-1933). Fue la época en la que la Educación llegó a ser cultural y políticamente santificada, siendo institucionalmente establecida como disciplina académica distintiva en las facultades de Filosofía de las universidades.

La institucionalización definitiva de las Ciencias de la Educación alrededor de 1920 finalizó un largo proceso que había empezado en 1779, cuando Ernst Christian Trapp (I745-1818), un ministro luterano y, así llamado, educador filantrópico, fue nombrado profesor de Filosofía y Pedagogía en la Universidad de Halle; su cátedra estaba localizada en la Facultad de Teología (luterana). La actividad educativa de Trapp era dirigir un instituto pedagógico para formar a profesores de escuela elemental. Sin embargo, este experimento no demostró ser muy sostenible ya que Trapp dejó la universidad alrededor de 1783 y fue reemplazado por un filólogo clásico: fue el amanecer del neohumanismo alemán con sus poetas y pensadores en torno a I800, alumbrando la auténtica cultura alemana en su esplendor, tal y como se cree hasta hoy, y fue la referencia a estos poetas y pensadores alemanes lo que permitió a la Pedagogía llegar a establecerse en las universidades alemanas como disciplina filosófico-teórica con su epítome de Bildung.

Muchos de los problemas institucionales con los que Trapp había estado lidiando fueron resueltos más de ciento treinta años antes, hacia el final de la Gran Guerra, cuando la Pedagogía fue ascendida como disciplina académica junto a las Humanidades (con preferencia sobre la Teología) y se centró en el entrenamiento de profesores del Gymnasium prospectivo (Escuela de gramática en el Reino Unido, academia-instituto en Estados Unidos), por encima de los maestros o profesores de escuela elemental. Y en contraste con la aproximación más práctica de Trapp, los nuevos profesores se centraron ahora en la Bildung y rechazaron aspectos de utilidad. La conservadora estructura y los inestables mecanismos de las universidades propiciaron una tradición sostenible de cultura tradicional, cuyos exponentes combaten hoy PISA con polémicas nociones enraizadas en una ideología antioccidental.

${ }_{12}$ La noción Bildung fue una noción polémica usada contra el desarrollo científico y económico en Francia e Inglaterra. Se popularizó hacia finales del siglo xvin y describía la exclusividad de la cultura alemana como oposición a la civilización occidental, ver también: HorLAcHER, Rebekka: Bildung, la formación, Barcelona, Octaedro, 2015. 
LOS FUNDAMENTOS RELIGIOSOS DE LA BILDUNG COMO EPÍTOME DE LA EDUCACIÓN ALEMANA Y SU FUNDAMENTAL RECELO ANTIOCCIDENTAL DANIEL TRÖHLER

Este artículo intenta proporcionar evidencias de cómo estilos de pensamiento específicos -definitivamente luteranos- permitieron la incorporación de la Pedagogía como disciplina universitaria en la República de Weimar, organizada intelectualmente en torno a la noción de Bildung, que excluyó aspectos prácticos en la concepción académica de la Pedagogía. Para ello se centrará en la época comprendida entre 1890 y 1925 , permitiendo tanto retrospectivas como prospectivas. En primer lugar, señalará que el destacable fenómeno del crecimiento económico, territorial y militar alemán hacia el final del siglo xIX no encontró estima en la inteligencia alemana debido a su fundamental filosofía dualista (sección r). Después se dirige al contexto cultural e institucional de esta filosofía dualista, en la cual se yergue la teoría de la Bildung y mantiene su atractivo (2). En una tercera aproximación se reconstruirá cómo las Ciencias de la Educación -basadas en la teoría de la Bildung- se convierten en disciplina académica durante la Gran Guerra (3). Y en un cuarto apartado se muestra cómo en el contexto de una autoadscrita misión mundial de la Persönlichkeit y Bildung se convirtieron en gritos por la batalla dominante contra Occidente y contra las ciencias empíricas (4). La sección final se centra en las medidas que los defensores de la teoría de la Bildung tomaron para asegurarle sostenibilidad y en cuáles han sido sus resultados hasta hoy (5).

\section{Crecimiento territorial, económico y militar y reivindicación de la naturaleza interna inmutable}

A diferencia de Inglaterra y Francia, Alemania se industrializó de forma manifiestamente tardía. Sin embargo, con la mecanización del procesamiento textil, la construcción de vías de tren, producción masiva de acero y las industrias electro-técnica y química rápidamente desarrolladas en el último cuarto del siglo XIx la industrialización provocó cambios fundamentales en la estructura social. Paralelamente a este crecimiento económico, Alemania se había expandido territorialmente en la Guerra Franco-Alemana en I870-71, cuando el nuevo imperio alemán (Deutsches Reich) bajo el liderato de Prusia, a su vez bajo el reino de la casa de Hohenzollern, se anexionó la Alsacia y la Lorena francesas.

Bajo el reinado del primer káiser alemán, Wilhelm I, y su canciller Otto von Bismarck, un crecimiento que todo lo abarcaba se produjo bajo una política de paz, ley y orden a grandes rasgos. Tras la muerte de Wilhelm I en i888, su hijo Frederick III asumió el poder, pero murió tan solo noventa y nueve días más tarde, dejando el trono a su hijo de veintinueve años Wilhelm II quien, cuando menos, tuvo una responsabilidad compartida en el estallido de la Gran Guerra en I9I4. Dos años más tarde de su coronación, en I890, el káiser Wilhelm II despidió al canciller Bismarck y empezó una nueva y agresiva política nacional. Desairó a la reaccionaria Rusia y como resultado los rusos formaron una alianza con la (tercera) República francesa. También desafió al poder naval británico expandiendo agresivamente la flota alemana. Y cuando Francia empezó a anexionarse el 
último territorio independiente en el norte de África, Marruecos, los alemanes protestaron con vehemencia, teniendo ello como consecuencia la Entente Cordiale entre Gran Bretaña y Francia. Hacia el final de la primera década del siglo xx con la excepción del simpatizante Imperio Austro-Húngaro, Alemania estaba más o menos aislada políticamente, pero se había convertido en la nación líder de Europa, económica y militarmente. Alemania era el único país europeo con más trabajadores industriales que agricultores, pero en política doméstica estaba retrasada: carecía ampliamente de democracia, milicia y estaba dominada por una improvisada alta burguesía terrateniente.

Detrás de la impresionante expansión y crecimiento, estaba nada menos que el sistema educativo que había sido establecido tras i87i con la ayuda de los pagos franceses en reparación por las pérdidas de guerra. Los maestros de la escuela elemental habían llegado a ser importantes agentes en el proceso de construcción de la nación y estaban nacionalmente entrenados en consecuencia; la historia de la Pedagogía y la Educación como asignatura jugó un papel crucial en el programa de entrenamiento, centrándose en los «héroes» alemanes ${ }^{13}$. Paralelamente al clásico Gymnasium (Escuela de gramática en el Reino Unido, academia-instituto en Estados Unidos), con énfasis en el latín y el griego, fueron establecidos otros dos modelos alrededor de 1850: el Realgymnasium sin un asomo de griego y con menos latín y más Matemáticas y Ciencias, y en torno a i88o, la Oberrealschule, sin griego ni latín y con más Matemáticas y Ciencias y con más lenguas extranjeras modernas en el currículum. Mientras que los graduados del clásico Gymnasium siguieron en las universidades alemanas más prestigiosas, muchos de los otros estudiantes entraron en los recién establecidos colegios técnicos (en Darmstadt en I877, Múnich en I877, Brunswick I878, Berlín en I879, Aquisgrán en I88o, Karlsruhe en 1885 , Stuttgart en I890 y Dresde en I890) ${ }^{14}$. Los colegios técnicos fueron un gran éxito: mientras que la adscripción de los estudiantes fue de sólo 2.242 en I870, había al menos cinco veces más estudiantes inscritos, esto es I0.59I, cuarenta años más tarde en $1910^{15}$.

Sin embargo, la expansión económica, militar, territorial y el sistema educativo no parecían proporcionar a los alemanes mucha autoconfianza. Fromkin, historiador de la Universidad de Boston, escribió que el creciente aislamiento político de su país en Europa molestaba a los alemanes:

La reclusión era la pesadilla de Alemania y Alemania lo ha atraído sobre sí misma. Localizada en el corazón de Europa, el país ha aterrorizado tan efectivamente a sus vecinos, que se han aliado juntos en autodefensa. Sucesivamente, lo que sus vecinos se han visto conducidos a hacer, ha reforzado largamente la paranoia alemana ${ }^{16}$.

I3 TRÖHLER, Daniel: «The formation and function of histories of education in continental teacher education curricula", Journal of the American Association for the Advancement of Curriculum Studies, 2 (2006). Disponible en: http://www.uwstout.edu/soe/jaaacs/vol2/trohler.htm.

${ }_{14}$ Wehler, Hans Ulrich: Deutsche Gesellschaftsgeschichte. Dritter Band. Von der 'Deutschen Doppelrevolution' bis zum Beginn des Ersten Weltkrieges I849-19I4, Múnich, C. H. Beck, I995, p. I225.

is Ibidem, p. I2II.

${ }^{16}$ Fromkin, David: Europe's last summer: Why the world went to war in 19I4, New York, Vintage, 2005, p. 96. 
LOS FUNDAMENTOS RELIGIOSOS DE LA BILDUNG COMO EPÍTOME DE LA EDUCACIÓN ALEMANA Y SU FUNDAMENTAL RECELO ANTIOCCIDENTAL DANIEL TRÖHLER

Fromkin detectó una contradicción fundamental que caracterizaba la situación alemana hacia ı900:

Culturalmente, la población de Europa más y mejor educada -la alemanase decía a sí misma que había sido sofocada por una civilización europea que la presionaba para ello desde todos los frentes. No era evidente entonces y tampoco ahora por qué los alemanes se sentían de esta manera, pero estaba claro que era como se sentían.

Una forma de entender esta contradicción es darse cuenta de que, en el marco de los valores culturales de una visión del mundo dualista, el progreso científico y tecnológico es aceptable, pero, al final, no es importante. La ideología se hace obvia con Rudolf Eucken, filósofo del nuevo idealismo de la vida (Lebensphilosophie) y ganador del premio Nobel de Literatura en 1908. Eucken reconocía que Alemania -como Francia, Inglaterra o América- había experimentado un tremendo crecimiento económico en el siglo xIX. La diferencia sustancial, de acuerdo con Eucken, sin embargo, era que este desarrollo no había corrompido el auténtico carácter de los alemanes:

¿Habíamos caído de nuestros propios estantes cuando nos volvimos hacia el mundo visible, cuando desarrollamos nuestras fuerzas en tierra y mar, cuando tomamos el liderazgo de la industria y la tecnología? ¿Habíamos, por tanto, negado nuestra auténtica naturaleza interior?... ¡No y otra vez no! ${ }^{17}$.

Esta auténtica naturaleza eterna, que, según Eucken, distinguía a los alemanes de todas las demás naciones ${ }^{18}$, es una vida espiritual interior que fue originalmente religiosa y que, a través del curso de la historia, llegó a caracterizar todo el pensamiento y la vida alemana. La Filosofía alemana, escribió Eucken, era esencialmente distinta de todas las otras filosofías; no era simplemente autoorientación en un mundo dado por sentado, sino más bien un atrevido intento de entender el mundo desde dentro de nosotros mismos; creó grandes corrientes de pensamiento, sistemas monumentales y con dichos sistemas intentó penetrar el mundo visible e incluso convertirlo en uno invisible ${ }^{\mathrm{r}}$.

Era este énfasis del mundo interior el que incluía una cierta indiferencia hacia el crecimiento económico y militar y el poder. Las clases dominantes en Alemania tenían pocas dudas de ser una nación líder, pero su liderazgo no debía ser

${ }_{17}$ Eucken, Rudolf: Die weltgeschichtliche Stellung des deutschen Geistes, Stuttgart, Deutsche Verlags-Anstalt, I9I4, p. 8.

${ }_{18}$ En el estallido de la Gran Guerra, no sólo los alemanes polemizaron contra los enemigos, sino que también los intelectuales británicos y franceses lo hicieron. Ver Wallace (1988) para el caso británico y Hanna (1996) para el francés. El German Philosophy and Politics de Dewey (I9I5) también se puede leer como polémica contra Alemania. Wallace, Stuart: War and the image of Germany: British Academics I9I4-19I8, Edinburgh, John Donald Publishers, I988. Hanna, Martha: The mobilization of intellect: French scholars and writers during the Great War, Cambridge, MA, Harvard University Press, 1996. Dewey, John: German philosophy and politics, New York, Holt, 1915.

19 EUCKen: op. cit., pp. I2 y s. 
LOS FUNDAMENTOS RELIGIOSOS DE LA BILDUNG COMO EPÍTOME DE LA EDUCACIÓN $\mathrm{I} 3 \mathrm{O}$ ALEMANA Y SU FUNDAMENTAL RECELO ANTIOCCIDENTAL

DANIEL TRÖHLER

derivado de maquinaria, armas o una economía afluente, sino del arte, cultura (alemanes) y de la Bildung. Eucken creía que este espíritu de vida interior se hacía tangible en el arte alemán y particularmente en la música, mucho más que en la tecnología ${ }^{20}$. Según Eucken, otro ganador del premio Nobel de Literatura, Thomas Mann, veía el arte de forma diametralmente opuesta al mundo exterior de la política y la alemanidad o el carácter alemán, como el equivalente al arte ${ }^{21}$. Música y carácter alemán de la mano con la música de Martín Lutero se habían convertido en una forma de moralidad ${ }^{22}$. Para Mann, el arte era la expresión de la Bildung, el cual, según él, era un término acuñado por uno de los héroes de los poetas y pensadores alemanes de los cuales había estado escribiendo Madame de Staël en I8ıo, llamado Goethe. La Bildung, decía Mann, era sólo para los alemanes ${ }^{23}$ y se refería a la cultivación, la formación de la vida interior espiritual del hombre ${ }^{24}$. En contraste con este ideal, Mann veía la democracia idéntica al materialismo o el capitalismo ${ }^{25}$ y atacó a los tres, señalando que la política en general era «impropia de Alemania» e incluso «hostil para Alemania» ${ }^{26}$. La democracia, siempre según Mann, se erigía como una contradicción a la Cristiandad y era traidora a la cruz ${ }^{27}$.

\section{El caldo de cultivo cultural e institucional de la teoría de la Bildung en torno a 1900}

De la misma forma que el crecimiento industrial no significó una reestructuración política - por ejemplo, hacia una mayor participación y democracia- la expansión masiva del sistema educativo y el compromiso a la Bildung no implicó una idea de oportunidades igualitarias para todos los niños. De acuerdo con la doctrina de la educación general de Humboldt (Allgemeinbildung) ésta debía ser estrictamente separada del entrenamiento profesional; la escuela elemental con su enfoque utilitario permaneció como un sistema autocontenido que era controlado por el clero. Los profesores de la escuela elemental no estaban formados en las universidades, sino en los separados y frecuentemente denominados seminarios de maestros (Lehrerseminare). Los requisitos de acceso para estos centros no incluían un diploma del Gymnasium (escuela superior de formación académica). En contraste, los estudiantes de las universidades prospectivas y los sirvientes de la administración civil pública no acudían a la escuela elemental, sino a un preescolar de tres años en su lugar (Vorschule), donde el «pre» significa pre-Gymnasium. Este pre-Gymnasium implicaba pagos sustanciales y de este modo seleccionó a las clases poderosas de las medias y bajas. Mientras que los aristócratas

Ibidem, p. I3.

TRÖHLER: 20I2, P. I53.

Ibidem.

23 De STAËL-Holstein, Anne Louise Germaine: De l'Allemagne, Paris, H. Nicolle, r8ıo, pp. 497 y s.

24 Ibidem, p. 249.

${ }_{25} \quad$ Ibidem, pp. 233, 346.

26 Ibidem, pp. 2I, 29, 256, 268.

27 Ibidem, p. 419. 
alemanes (cada vez más empobrecidos), la milicia y la alta burguesía incrementaban su dominio de la administración pública y ambas coincidían en que el nuevo dinero -capitalismo- era de clase baja.

La manera en la que la burguesía alemana interpretó y favoreció la educación general (Allgemeinbildung) como estrictamente opuesta a la formación vocacional (Berufsbildung) resulta evidente en la ecualización de los tres tipos diferentes de Gymnasium que posibilitaban el acceso a las universidades. Hasta ı9oo, sólo el diploma del Gymnasium clásico, con el estudio del latín y el griego, proporcionaba acceso ilimitado a las universidades. Había restricciones respecto a los diplomas del Realgymnasium (con latín, sin griego y con más ciencias) y sobre todo de la Oberrealschule (sin latín ni griego y con más ciencias y más lenguas modernas extranjeras). La Asociación de Ingenieros Alemanes, la Asociación de los Científicos Naturalistas y Físicos alemanes y la Asociación de Químicos Alemanes ejercían presión para hacer equivalentes los distintos tipos de Gymnasium y en una conferencia escolar en I890, el káiser Wilhelm II (I89I) pidió la abolición del Realgymnasium y un enfoque mayor en la Historia de Alemania y la lengua:

Cualquiera que haya estado en un Gymnasium por sí mismo y haya echado un vistazo tras la escena, sabe qué falta. Una base nacional espera. Los fundamentos del Gymansium deben ser alemanes; debemos criar jóvenes alemanes nacionales y no jóvenes griegos y romanos ${ }^{28}$.

Los interesados en conservar el Gymnasium clásico (con latín y griego) reaccionaron prontamente fundando el alemán Gymansialverein (Asociación Alemana de Gymnasium) para defender la supremacía del Gymnasium clásico. Los profesores del Gymnasium obtuvieron mucho apoyo en su batalla por conservar miembros en las facultades de las universidades, donde se oponían a tratar con estudiantes a los que les faltaran profundas competencias en latín y griego; y fueron respaldados por las preferencias culturales de la burguesía, que había completado el Gymnasium clásico: en I900, el año en que los tres tipos de Gymnasium llegaron a ser equivalentes, el $82 \%$ de los estudiantes tenía un diploma del Gymnasium clásico, el 12,5\% del semiclásico Realgymnasium y sólo el 5,5\% de la Oberrealschule. Catorce años más tarde las proporciones habían cambiado, pero la abrumadora supremacía del Gymnasium clásico prevalecía: en 1914, el 60\% tenía un diploma del Gymnasium clásico, el 24\% del semiclásico Realgymnasium y sólo el i6\% de la Oberrealschule ${ }^{29}$.

No hay duda alguna de que el clásico Gymnasium era el cuadro de unidad de formación de una clase superior con pocos derechos políticos. No obstante, ya que el área política se veía como antialemana, el foco estaba mucho más centrado en el ámbito de la vida interior, en la Bildung. El currículum del Gymnasium revela los ingredientes de este constructo cultural:

28 TRÖHLER: 2OI2, p. I54.

29 Becker, Hellmut y Kluchert, Gerhard: Die Bildung der Nation, Berlin, J. G. Cotta, 2002, p. I3. 
LOS FUNDAMENTOS RELIGIOSOS DE LA BILDUNG COMO EPÍTOME DE LA EDUCACIÓN

Figura i: Mayoría de asignaturas de la escuela en el Gymnasium clásico ${ }^{\circ}$

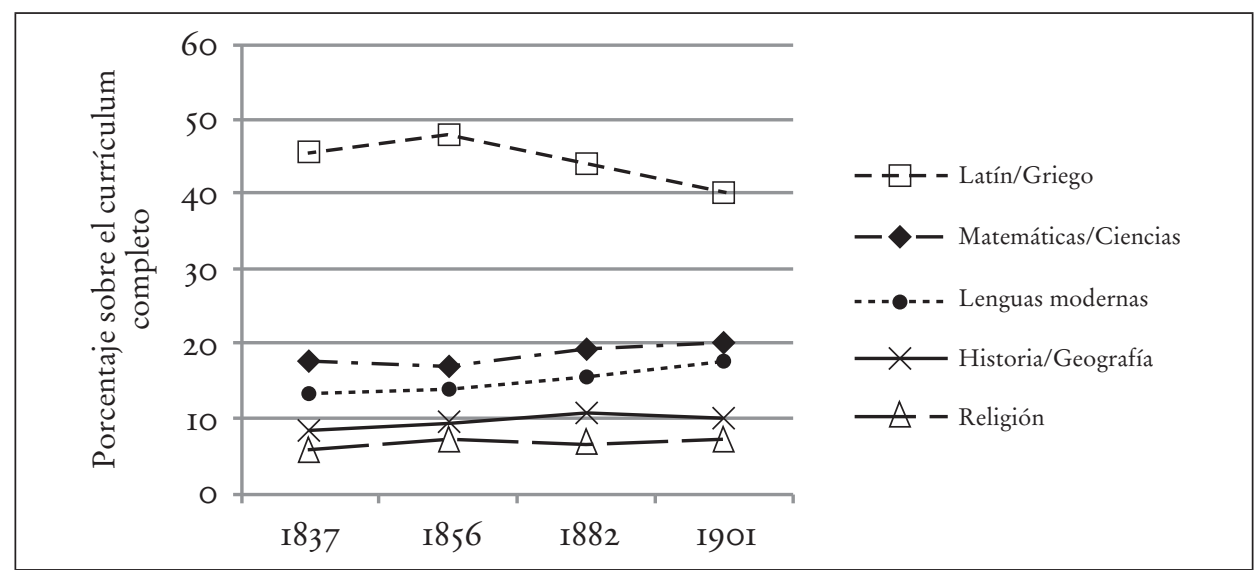

Hasta 1900, a más de la mitad de estudiantes (80\%) del Gymnasium (representan menos del $5 \%$ de un grupo de edad) y, por tanto, a la probable clase alta alemana se le impartían las asignaturas de la escuela como sigue: bastante por encima del $40 \%$ de la clase se dedicaba al griego y al latín, casi el $20 \%$ del tiempo de clase a las Matemáticas y las Ciencias, algo menos del 20\% del tiempo de clase a las lenguas modernas (sobre todo, alemán), alrededor del ro\% de la clase a Historia y Geografía y algo menos del ro\% del tiempo de clase a la Religión.

Indudablemente, durante el siglo xIx, el latín y el griego habían perdido algunos puntos de porcentaje, ganados por la lengua alemana. El énfasis en la lengua y la historia alemanas ${ }^{3 \mathrm{I}}$ hacia final de siglo no debilitó el pretendido mundo ideal de la Antigüedad, pero ayudó a solidificar la idea de que la cultura alemana era la verdadera heredera de los griegos y, por tanto, el modelo de la Antigüedad presente, y la mayoría de la Antigüedad griega, permanecía como un modelo inigualable, pero los alemanes lo creían y tenían una ventaja sobre todas las otras naciones de su tiempo: la lengua alemana. Volviendo a I808, cuando Alemania estaba ocupada por las tropas francesas y Madame de Staël alababa a los poetas y pensadores alemanes por encima de los franceses, sin que esta alabanza quedara exenta de polémica, fue el renombrado idealista alemán Johann Gottlieb Fichte (I808/2009) quien en su famoso Discursos a la nación alemana había proclamado la necesidad de una «completa reforma del sistema educativo actual y el único medio de preservar la existencia de la nación alemana» ${ }^{32}$. Esta educación no se

Fuente Herrlitz, Hans-Georg; Hopf, Wulf y Titze, Harmut: Deutsche Schulgeschichte von I800 bis zur Gegenwart. Eine Einführung, Weinheim, Juventa, I993, p. 68.

${ }^{31}$ RoHLfes, Joachim: «Deutscher Geschichtsunterricht im I9. Jahrhundert: Staatlich-politische Vorgaben, geschichtswissenschaftliche Maßstäbe, pädagogische Impulse», Geschichte in Wissenschaft und Unterricht, 54 (2004), pp. 382-400.

${ }_{32}$ Fichte, Johann Gottlieb: Addresses to the German nation (G. Moore, Ed. and Trans.), Cambridge, England, Cambridge University Press, 2009, p. 17 (Trabajo original publicado en alemán en I808) (Discursos a la nación alemana, Madrid, Tecnos, I988). 
centraba en el aprendizaje y las ciencias modernas, sino que consistía en una parte intelectual-espiritual (geistig) y una parte emocional. En la parte intelectualespiritual los hombres jóvenes debían ser liderados para reconocer el arquetipo de una buena vida y en la parte emocional debían hacerse fuertes para implantar estos arquetipos en la vida real ${ }^{33}$. Este arquetipo no era empírico sino metafísico: «Por tanto, en su mismo inicio, esta educación alumbra un conocimiento que sobrepasa verdaderamente toda experiencia, que es súper-sensible, estrictamente necesario y general, que abraza por adelantado todas las posibles experiencias subsiguientes» ${ }^{34}$. Los alemanes creían tener un acceso privilegiado a estos arquetipos debido precisamente a la pureza de la lengua alemana, que permitía reconocer la esencia interior de las cosas. En este contexto Fichte afirmó, como hizo el sociólogo Sombart cien años más tarde (ver más abajo), que de hecho los alemanes entendían al extranjero mejor de lo que lo hacía él mismo, mientras que el extranjero no entendía al «auténtico alemán»35.

Es por consiguiente la lengua alemana la única lengua pura que permite reconocer las verdades universales, no distorsionadas por la experiencia, la que motiva a reconocerlas ${ }^{36}$, «educando al ser humano perfecto», reconociendo el «estado perfecto» ${ }^{37}$, abarcando, empezando por suelo alemán, una nueva época «en la que la raza humana se desarrolle a propósito y libremente» $3^{8}$. Mientras que la vieja (y mala) educación tenía que motivar a los jóvenes a aprender prometiendo «que este aprendizaje sería útil en el futuro», la auténtica educación no buscaba ser «sierva de la propia sensación de bienestar», ya que esto habría producido gente inmoral ${ }^{39}$. Esta es la clásica visión luterana dualista del mundo, que favorece uno de los polos de esta dualidad y reconocemos que aún hoy permanece en los argumentos que esgrimen los críticos alemanes del informe PISA.

La perfección interior de la Bildung fue el lema o el epítome de la alemanidad como opuesta a cualquier otra región del mundo. Esta idea regresa al debate luterano de la perfección del alma, siendo el alma en el protestantismo el modelo en la tierra que permite a los hombres conexión directa con Dios (y no mediada por una institución como la sagrada madre Iglesia). A final del siglo xvir había sido el teólogo luterano, filósofo y abogado Samuel von Pufendorf quien había explicado en su trabajo acerca de la ley natural la conexión entre el hecho del alma humana, la razón y la dignidad humana: «El hombre es de la más alta dignidad porque tiene un alma la cual es distinguible por la iluminación de la razón, por la habilidad para juzgar cosas y para decidir libremente y que está familiarizada con muchas artes $»^{40}$. Esta idea protestante fue desarrollada en mayor

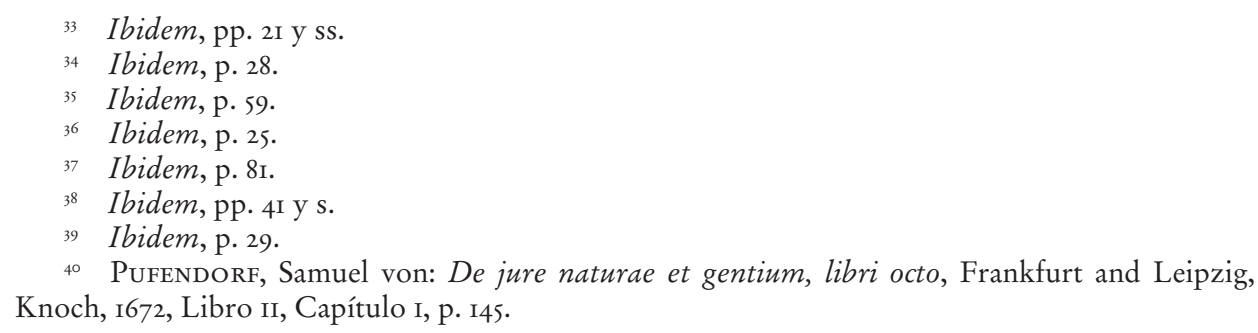


LOS FUNDAMENTOS RELIGIOSOS DE LA BILDUNG COMO EPÍTOME DE LA EDUCACIÓN

profundidad, entre otros, por el teólogo luterano, filósofo y abogado Christian Wolff. En su trabajo seminal Jus naturae methodo scientifica per tractum ${ }^{41}$, Wolff defendió la igualdad y la dignidad de los hombres partiendo del hecho de que todos los hombres habían sido creados por Dios y tenían, por tanto, una disposición natural a la perfección -que los distinguía del resto de los seres de la tierra ${ }^{42}$. Según Wolff, la perfección es un elemento antropológico, tal y como diríamos hoy en día, independientemente del grado en el que un hombre concreto la realice en su vida. Wolff distingue por consiguiente una perfección esencial o natural (perfectio essentialis) del hombre de un estado accidental de realización de la perfección (perfectio accidentalis) que los hombres concretos pueden poner en práctica en sus condiciones de vida ${ }^{43}$. Immanuel Kant se hace eco de la formulación de esta idea de esta forma: cualquier ser humano tiene un «carácter, el cual crea él mismo, hasta donde es capaz de perfeccionarse a sí mismo en función del fin que él mismo adopte»44. La base esencial en su «capacidad de razón (animal rationabile)» permite a los hombres «hacer de sí mismos un animal racional». La vía (educacional) a esta perfección interna es el proceso de la Bildung, cuyo resultado es poseer Bildung y su materialización es la Persönlichkeit.

\section{Defensa nacional doméstica en 19I7: la entrada de la Pedagogía como materia universitaria}

La idea de que los alemanes no eran apreciados (a pesar de su supremacía cultural) persistió a lo largo de todo el siglo xix y creció de manera dramática en el estadillo de la Gran Guerra en I9I4. Representante de la inteligencia alemana, el sociólogo Werner Sombart lo reivindicaba de acuerdo con Fichte (ver más arriba):

Entendemos a todos los extranjeros, aunque ellos no nos entiendan, y ninguno pueda entendernos... Ellos no nos entienden pero sienten nuestra vasta superioridad espiritual-intelectual (geistig)... Así que permitid a los alemanes de nuestro tiempo caminar sobre el mundo orgullosamente, con las cabezas en alto en el seguro entendimiento de que nosotros somos el pueblo de Dios ${ }^{45}$.

Había algunas ventajas en esta falta de comprensión, afirmó el filósofo Max Scheler: en cuanto a los británicos, pese a haber robado patentes alemanas en

${ }_{41} \quad$ Ley natural, tratada según el método científico.

42 WolfF, Christian von: Theologia naturalis, method scientifica per tractata. Pars sexta, Frankfurt y Leipzig, Libraria Rengeriana, 1746, p. 592.

${ }_{43}$ WolfF, Christian von: Theologia naturalis, methodos cientifica per tractata. Pars prior, Frankfurt y Leipzig, Libraria Rengeriana, 1738, Capítulo III, 579, 530.

44 KANT, I.: «Anthropology from a pragmatic point of view», en The Cambridge Edition of the Works of Immanuel Kant-Immanuel Kant: Anthropology, history, and education, Cambridge, England, Cambridge University Press, 1798/2007, pp. 227-429, 417.

45 Sombart, Werner: Händler und Helden: Patriotische Besinnungen, Munich, Duncker und Humblot, 1915, pp. I32 y ss. 
tecnología, su principio utilitarista no tenía acceso al espíritu implicado en las patentes alemanas ${ }^{46}$. Y el filósofo y educador Eduard Spranger añadía: que «la Física y las tecnologías estaban muertas si no se despertaba el espíritu que las había creado» ${ }^{47}$. Las naciones occidentales fueron incluso acusadas de democracia malentendida, tal y como dijo el filósofo neokantiano Paul Natorp:

La Democracia significa para los alemanes algo completamente distinto que para todos los demás. Pueblo, demos, es para nosotros no pluralidad, la cual prevalece a través de la suma mecánica de sus energías sociales...; pero pensamos en él como en la totalidad de los miembros de la comunidad (Volksgenossen), en la cual tenemos la mejor y la máxima confianza ${ }^{48}$.

La percepción de ser el pueblo elegido era una noción de lugar común. Eucken, el ganador del premio Nobel mencionado más arriba, etiquetó a los alemanes como «alma de la humanidad». Proclamando en referencia a Fichte la significación histórica del mundo para los alemanes ${ }^{49}$ y explicando la diferencia entre el ideal griego y el ideal alemán: el ideal griego, escribió Eucken, trataba el mundo tal y como venía dado, mientras que el alemán veía el mundo como algo que llegará a ser y tomaba responsabilidad en su posterior desarrollo:

Queremos intervenir, mejorar, promover; damos a la Historia un profundo significado. Mientras que el ideal griego era primordialmente artístico, el alemán representaba un idealismo ético. Los antiguos alababan la contemplación pero para nosotros es la acción lo que cuenta, la acción del Persönlichkeit, el acto del mundocreador y el mundo-formador ${ }^{\text {so }}$.

En este contexto de mundo-misión, había que plantearse la cuestión de si la Pedagogía y o las Ciencias de la Educación, con énfasis en la Bildung, debían convertirse o no en un campo de estudio autónomo en las universidades. En un interesante cierre de filas, el aristócrata conservador August von Trottzu Solz, quien era ministro alemán de Educación y Artes; Ernst Troeltsch, teólogo alemán y filósofo cultural, y Eduard Spranger, joven profesor de Filosofía y Educación en la Universidad de Leipzig, abrieron la discusión sobre el futuro de las Ciencias de la Educación como materia de las universidades con ocasión de la conferencia de Educación que tuvo lugar en Berlín en mayo de i917. La idea ya había sido concebida por Eduard Spranger en I9I5, cuando envió a August von Trottzu Solz un memorando sobre los estudios relacionados con la Educación

\footnotetext{
${ }_{46}$ Scheler, Max: Die Ursachen des Deutschenhasses: Eine nationalpädagogische Untersuchung, Leipzig, Kurt Wolff Verlag, I917, pp. 78 y s.

47 Spranger, E.: «Von der ewigen Renaissance (I9I6)», en Spranger, E.: Kultur und Erziebung. Gesammelte pädagogische Aufsätze, Leipzig, Quelle \& Meyer, 1919, pp. I32-I5I, I50.

$4^{8}$ Natorp, Paul: Deutscher Weltberuf. Band 2: Die Seele des Deutschen, Jena, Eugen Diederichs, I918, p. I3I; traducción libre.

49 EuCKen: op. cit., pp. 22 y s.

so Ibidem: pp. 20 y s.
} 
en la Universidad ${ }^{\text {s }}$. En el memorando, Spranger expresaba su convicción de que tras la guerra la educación de la juventud alemana sería tan importante para la nación alemana como en ese momento lo era el Estado Mayor. Spranger advirtió que el interés fundamental de la nación en la educación podría ser pobremente dirigido si la Educación no llegaba a ser un campo de estudio académico en la universidad $^{52}$. Sin embargo, la educación en la universidad «no debía ser demasiado práctica», enfatizaba Spranger, ya que toda la educación universitaria debía centrarse en conexiones generales y elementos educativos en su conjunto ${ }^{53}$.

El memorando de Spranger refleja su socialización cultural e intelectual en el Gymnasium clásico al que había acudido en sus estudios de Filosofía, Historia y alemán en la Universidad de Berlín (1900-1905). Tras completar su doctorado en fundamentos de Historia en 1905, Spranger siguió trabajando en su tesis centrada en la idea del humanitarismo en la obra de Wilhelm von Humboldt ${ }^{54}$. De acuerdo con ello, la Educación a nivel académico no habría de ser práctica sino filosófica o cultural-filosófica (kulturphilosophisch), y debería servir a la política educacional alemana, conservadora y antisocialista, que había prevalecido siempre desde que el nuevo káiser Wilhelm II fue coronado en i888. Esta tendencia conservadora en el memorando de Spranger encajó bien en la agenda del Ministerio alemán de Educación y Artes en 1917, cuando llegó a ser evidente que la guerra no se ganaría en unos pocos meses, como se había pensado en i9i4. Imbuido del asunto educativo como había estado el discurso nacional después de i89o, una reforma del sistema educativo parecía necesaria cuando se hizo evidente que la juventud alemana en los campos de batalla europeos se mostraba demasiado débil para derrotar a los inferiores ejércitos de los enemigos.

Las universidades apoyaron la introducción de la Educación en un campo autónomo de estudio que no fuera experimental, empírico o psicológico. Ernst Troeltsch, quien representaba a las universidades en la conferencia de Berlín en mayo de 1917, recordó en sus palabras de clausura que la Educación no debería estar construida desde la Psicología, sino desde la Historia (de la enseñanza), desde instituciones educativas y desde la Filosofía cultural. Troeltsch advirtió que detrás de la Psicología había una oculta demanda igualitaria de igualdad de oportunidades en la educación y, por tanto, de una elevación utilitaria de las masas. «Esta es la verdad sobre la Psicología; que busca liderazgos en cualquier parte, es una metafísica oculta y una doctrina utilitarista del progreso»s5. «Utilitarismo»

$\because \quad$ Tenorth, H.-E.: «Pädagogik für Krieg und Frieden: Eduard Spranger und die Erziehungswissenschaft an der Universität Berlin von I9I3-I933», en Horn, K.-P. y KemniTz, H. (eds.): Pädagogik Unter den Linden: Von der Gründung der Berliner Universitätt im Jabren I8Io bis zum Ende des 20. Jahrbunderts, Wiesbaden, Franz Steiner Verlag, 2002, pp. 191-226, 204.

${ }_{52}$ Ibidem.

$\$ 3$ Ibidem, pp. 204 y s.

${ }_{54}$ SPRANGER, Eduard: Wilhelm von Humboldt und die Humanitätsidee, Berlín, Verlag von Reuther \& Reichard, 1909.

"Troeltsch, 1917, según se cita en Drewek, Peter: «Die Herausbildung der "geisteswissenschaftlichen” Pädagogik vor 1918 aus sozialgeschichtlicher Perspektive», Zeitschrift für Pädagogik, supl. 34 (1996), pp. 299-315, 301. 
fue la maldición de los filósofos y educadores de la cultura protestante de mentalidad nacionalista, como se puede ver en una carta de Eduard Spranger escrita a Georg Kerschensteinerin, en 1915, en la cual desprecia el pragmatismo de Dewey tachándolo de «utilitarismo de cocina y mantenimiento» $\$$.

Con todo ello, los problemas que Trapp había enfrentado en 1779 estaban resueltos. La educación en la universidad no era práctica, sino teórica, no dirigida a los maestros de escuela elemental o profesores de la escuela normal, sino a profesores de formación académica en la escuela superior (profesores de Gymnasium) y estaba emancipada de la Teología y orientada hacia la Filosofía (protestante). En contra de este contexto, los maestros de educación elemental, que deseaban formarse en la universidad, eran rechazados (ya que esta formación era práctica), y la Educación o la Pedagogía se convirtió en una disciplina académica en las universidades, dirigida únicamente a la formación de los futuros profesores de Gymnasium. Las nuevas regulaciones de evaluación anticipaban exámenes de tipo científico en las asignaturas de la escuela que los futuros profesores de Gymnasium fueran a impartir, con exámenes de tipo educativo donde demostraran una habilidad susceptible de ser reflejada en problemas educativos. Filosofía era el único curso que era requisito para los prospectivos profesores de Gymnasium, no importaba si posteriormente enseñaban Química, Historia o Físicas7. La formación universitaria de los aspirantes a profesores de Gymnasium estaba, por tanto, dominada por la Filosofía cultural nacional(ista) y dualista, la lealtad al káiser y el apoyo a la sociedad de clases. El modelo de Educación admitido en las universidades en I9r7 debía seguir estas líneas, y así lo hicieron sus principales actores mediante la elaboración de la geisteswissenschaftliche Pädagogik durante la República de Weimar.

\section{La misión alemana. Persönlichkeit y Bildung}

Alemania sufrió una derrota contra el poder aliado en la Gran Guerra. Alrededor de dos millones de soldados alemanes fueron asesinados y más de ochocientos mil civiles murieron de hambre. El descontento con el káiser Wilhelm II creció y cuando, en noviembre de 1918, la Revolución alemana de inspiración socialista estalló, el káiser Wilhelm II y la nobleza abdicaron. Un alto el fuego terminó la guerra el ir de noviembre y se fundó la República alemana. Los desacuerdos entre la izquierda -especialmente entre los marxistas radicales y los socialdemócratasevitaron que Alemania siguiera el camino que había tomado Rusia. Tras la firma del Tratado de Versalles en junio de 1919, se escribió y adoptó la Constitución de Weimar en agosto de igr9 y se introdujo por primera vez la democracia entre las disputas alemanas.

56 Spranger, E.: [Letter sent to Georg Kerschensteiner on 22 March 1915], en Englert, L. (ed.): Georg Kerschensteiner. Eduard Spranger. Briefwechsel I9I2-1931, Oldenburg, Teubner Verlagsgesellschaft, 1966, pp. 35-38, 37 .

57 «Ordnung der Prüfung für das Lehramt an höheren Schulen in Preussen», en Zentralblatt für die gesamte Unterrichtsverwaltung in Preussen, Berlin, J.G. Cotta, 1917, pp. 613-647. 
La reconstrucción de Alemania estaba en juego, pero se disputaba el significado de esa reconstrucción. De un modo interesante y representativo, los intelectuales no dieron por sentadas estas disputas políticas, a pesar de su vehemencia. Ernst Troeltsch, mencionado más arriba, reconoció las disputas, pero trascendieron de un modo que parecían permanecer unidas contra el mundo occidental. En 1923 Troeltsch escribió Naturrecht und Humanität, que trataba de la ley natural y el humanitarismo en la política mundial. A pesar de la «fuerte fragmentación mental-espiritual», reconocía una «unidad mental-espiritual» que no podía -vemos nuevamente los mismos argumentos- ser entendida por ningún extranjero ${ }^{\varsigma 8}$. La razón estaba en que más allá de los argumentos y las preferencias políticas, los alemanes compartían «un modo de pensar político, histórico y moral alemán» que Troeltsch vio como opuesto al «modo de pensar de Europa occidental y América» ${ }^{9}$. Este último se entendía como un descendiente de la moderna ley natural social y políticamente interpretada, mientras que el pensamiento alemán era visto como el «romanticismo contra la revolución» ${ }^{60}$, como la encarnación de la estricta ley natural interior moderna. El pensamiento occidental se basaba en la asunción atómica igualitaria de la materia y la sociedad y de la ley natural universal. Mientras que el pensamiento alemán se basaba en una armoniosa Persönlichkeit ${ }^{6}$. Troeltsch veía a las naciones occidentales como el resultado de la moderna ley natural politizada, como la Declaración de los Derechos Humanos, e ignoraba los distintos fundamentos políticos de Estados Unidos y Francia, por ejemplo, para defender la idea del Romanticismo alemán y su superioridad ${ }^{62}$. Troeltsch rechazaba en particular la idea occidental de derechos igualitarios entre sus ciudadanos y reivindicaba que los alemanes habían superado este concepto superficial y, por tanto, habían creado los fundamentos para construir una nación basada en la responsable y autónoma Persönlichkeiten para asegurar un «extraordinario progreso a la moralidad libre, personal e individual» ${ }^{63}$.

Entre otros, dos filósofos más bien jóvenes con un fuerte interés en la Educación se comprometieron a establecer una teoría educacional o teoría de Bildung que encajara en esta ideología nacionalista y antioccidental. Ambos habían ido al mismo Gymnasium clásico en Berlín bajo la dirección de Ludwig Bellermann, un profesor de Filología clásica y alemana, especializado en Sófocles y Friedrich Schiller (otro héroe de los poetas y pensadores alemanes de alrededor de i8oo). Uno de ellos era Eduard Spranger (I882), arriba mencionado, que fue nombrado profesor de Filosofía y Educación en el Berlín de 1920 y el otro era Herman Nohl (I879), quien era tres años mayor que Spranger y había completado su habilitación bajo la tutela del arriba mencionado Rudolf Eucken en 1908 y fue nombrado

58 Troeltsch, Ernst: Naturrecht und Humanität, Berlín, Verlag für Politik und Wirtschaft, I923, p. 4.

59 Ibidem.

60 Ibidem, p. 6.

${ }_{61}$ Ibidem.

${ }_{62}$ Ibidem, pp. I3 y s.

${ }_{3}$ Ibidem, pp. 21 y s. 
profesor de Pedagogía en Göttingenin en 1922. Junto al alumno de Nohl, Wilhelm Fitner (I889), constituyeron la geisteswissenschaftliche Pädgogik para servir a los ideales predominantes de la inteligencia durante la República de Weimar ${ }^{64}$.

La construcción de la teoría de la Bildung implicó distintos elementos ${ }^{65}$. En primer lugar, los tres hombres repitieron que el mundo occidental no entendía la misión alemana. Con ocasión del memorial del fundador del Reich alemán en I87I, Spranger habló sobre la influencia del neohumanismo (los poetas y pensadores alemanes de alrededor de I800) en el amanecer de la conciencia nacional alemana. Spranger enfatizó que nadie en el mundo había sufrido tanta injusticia como estaban sufriendo los alemanes en aquel momento con el Tratado de Versalles: «incomprensión de todos los demás pueblos». Alemania tenía que soportar todas las injustas consecuencias de la Gran Guerra ${ }^{66}$, y era el momento de adherirse a los fundamentos de la nación alemana y su peculiar carácter, donde «alemanidad, helenismo y humanidad» parecían ser «una gran identidad» ${ }^{67,68}$.

En segundo lugar, se reclamó una Bildung mental-espiritual como única para el carácter alemán. Spranger escribió que desde Lutero hasta Fichte los alemanes habían trabajado en una idea: «Un proceso eterno, un proceso para alcanzar la mayor altura» ${ }^{69}$. A título individual, Spranger explicaba en 1928 que este proceso se denominaba Bildung y que los modelos de este Bildung eran «nuestros clásicos» -esto es, los poetas y pensadores alemanes en torno a $18000^{70}$. El significado de Bildung no es arbitrario sino eterno y conduce a la Personalität, la formulación de Spranger de la noción de la Persönlicbkeit. Ciertamente los griegos ayudaban a entender esta idea, pero según Spranger fueron los alemanes de alrededor de i8oo los que la desarrollaron por completo y le dieron identidad nacional ${ }^{7}$.

${ }^{64}$ Agradezco el meritorio trabajo que Klaus-Peter Horn ha realizado recolectando todos los datos relevantes de la educación escolar alemana en el siglo xx e interpretándolos en una ciencia de la historia de la educación en Alemania. HorN, Klaus-Peter: Erziehungswissenschaft in Deutschland im 20. Jabrbundert: Zur Entwicklung der sozialen und fachlichen Struktur der Disziplin von der Erstinstitutionalisierung bis zur Expansion, Bad Heilbrunn, Klinkhardt, 2003.

6s Para más detalles, sobre la geisteswissenschaftliche Pädagogik alemana y su epítome en la Bildung, ver: TröHLer, D.: «La geisteswissenschaftliche Pädagogik alemana y la ideologie de la Bildung», en TröHLER, D.: Los lenguajes de la educación. Los legados protestantes en la pedagogización del mundo, las identidades nacionales y las aspiraciones globales, Barcelona, Octaedro, 20I3b, pp. 177-192.

${ }_{66}$ Spranger, Eduard: Der Anteil des Neubumanismus an der Entstebung des deutschen Nationalbewusstseins, Berlín, Norddeutsche Buchdruckerei, 1923, p. 3.

${ }^{67}$ Ibidem, p. 6.

${ }_{68}$ Durante la Gran Guerra, el tropo de la incomprensión hacia los alemanes se había interpretado como odio hacia los alemanes, ver Magnus Hirschfeld (1915, ¿ Por qué nos odian los pueblos?) o Max Scheler (1917, La causa de la alemanofobia). Hirschfeld, Magnus: Warum hassen uns die Völker?, Bonn, Marcus \& Weber Verlag, 1915. Scheler, Max: Die Ursachen des Deutschenhasses: Eine nationalpädagogische Untersuchung, Leipzig, Kurt Wolff Verlag, 1917.

69 SPRANGER: I923, p. II.

7o Spranger, Eduard: Der deutsche Klassizismus und das Bildungsleben der Gegenwart (2. ${ }^{\mathrm{a}}$ ed.), Erfurt, Verlag Kurt Stenger, 1928, p. II.

${ }_{71}$ Ibidem, p. 22. 
En tercer lugar, estaba la demanda de unidad y armonía. En consonancia, el canon tradicional de asignaturas de la escuela no se creía útil para reconstruir el sistema educativo. Para la (no muy valorada en realidad) escuela elemental, Spranger promovía la asignatura de Historia local y Geografía (Heimatkunde), porque en Heimatkunde los estudiantes podían «comprender las raíces naturales y existenciales de nuestra existencia $»^{72}$ : «La educación humanística alemana tenía que empezar con la patria (Heimat), un método para iniciar a los niños en la lengua alemana, la historia alemana, competencias en alemán, en resumen, la cultura alemana» ${ }^{73}$. El estilo alemán no era plural sino uniforme, no era variado sino total, de modo que la historia y la geografía locales eran adecuadas para la reconstrucción de la derrotada nación. Era la asignatura la que enseñaba la «totalidad de las relaciones naturales y espiritual-mentales del ser humano, el más puro ejemplo de ciencia totalizadora», la «escuela totalizadora es el significado que necesitamos para superar la lucha interior y exterior del presente» ${ }^{74}$.

Siguiendo a Spranger, su compañero Nohl75 (1926) argumentó que todas las ideas actuales de reforma de la educación en Alemania -movimiento de reforma de la educación artística, movimiento de reforma de la educación vocacional, movimiento de la juventud, etc.- eran fragmentarias y controvertidas. No obstante, continuaba Nohl, si había algo que valiera la pena en estos movimientos «entonces debe ser una unidad final, de la que derivaran su mejor significado y vitalidad: la unidad de un nuevo ideal de la persona alemana y de un folclore mental-espiritual superior ${ }^{76}$. Nohl rechazó explícitamente verbalizar estos movimientos, ya que «esto causaría disputas», obviando el ideal y la «voluntad de elevar a vida a través de la espiritualización y la fe, dado que existe un significado más profundo en el mundo» ${ }^{77}$. Todos los movimientos de reforma compartían la idea de una «nueva Paideia de nuestro pueblo a una vida espiritual-mental elevada y uniforme en una comunidad elevada» ${ }^{78}$. Siguiendo esta idea, la nueva educación académica tenía que entender todas las expresiones verbales, prácticas e institucionales de la educación con el objetivo de guiar estas actividades hacia la unidad y la totalidad 79 . La totalidad estaba desdoblada en dos y ambas partes eran mutuamente dependientes: la totalidad del pueblo y la totalidad de la Persönlichkeit llamado Bildung ${ }^{80}$. O tal y como lo expresaba Nohl:

72 Spranger, Eduard: Der Bildungswert der Heimatkunde (2. ${ }^{a}$ ed.), Leipzig, Reclam, 1943, p. 6 (primera edición publicada en I923).

73 SPRANGer, Eduard: «Grundlegende Bildung, Berufsbildung, Allgemeinbildung», Preussische Fortbildungsschulzeitung, 9 (1918), pp. 65-77, 67.

74 SPRANGer: 1943, pp. 22 y s., 33, 43.

75 Nohl, Herman: «Die Einheit der Pädagogischen Bewegung», Die Erziehung. Monatsschrift für den Zusammenhang von Kultur und Erziebung in Wissenschaft und Leben, I (1926), pp. 57-6I.

${ }^{76}$ Ibidem, pp. 57 y s.

77 Ibidem, pp. 59 y s.

${ }_{78}$ Ibidem, p. 6I.

79 Ibidem.

8o SPRANGER: 1943, p. 6. 
LOS FUNDAMENTOS RELIGIOSOS DE LA BILDUNG COMO EPÍTOME DE LA EDUCACIÓN ALEMANA Y SU FUNDAMENTAL RECELO ANTIOCCIDENTAL

DANIEL TRÖHLER

Bildung es el subjetivo arte de la cultura, la forma interna y la estancia mentalespiritual del alma, que asimila, mediante su propio esfuerzo, los estímulos externos para una vida interior uniforme y capaz de formar cualquier expresión y acto de esta vida interna uniforme ${ }^{81}$.

\section{La Bildung en el imperio educativo: Geisteswissenschaftliche Pädagogik}

La educacionalización de la misión nacional alemana y su coherencia con la dominante ideología elitista antioccidental de los interesados políticos e intelectuales trajeron una modalidad particular de ideología educativa a las universidades alemanas y éstas desarrollaron estrategias para asegurar su sostenibilidad. Una de estas medidas fue dar voz a los estudiantes de doctorado en una serie de publicaciones fundada en 1923 y editada por Herman Nohl, las Göttinger Studienzur Pädagogik (Estudios de Göttingen sobre Pedagogía), publicada por la prestigiosa editorial Julius Beltz. Otra medida fue convocar a algunos estudiantes de Filosofía y Pedagogía para fundar el periódico Die Erziehung - Monatsschrift für den Zusammenhang von Kultur und Erziehung in Wissenschaft und Leben (La Educación - Periódico mensual para la interrelación entre Cultura y Educación en Ciencia y la Vida) en 1926, que fue publicado por la igualmente prestigiosa editorial Quelle \& Meyer en Leipzig. La tercera medida fue el intento de canonizar la teoría en un Manual de Pedagogía de cinco volúmenes que Nohl editó junto con Ludwig Pallat ${ }^{82}$; el editor fue nuevamente Julius Belz. Y la cuarta medida fue una eficaz política de adjudicación de plazas universitarias según la cual se obstaculizara al profesorado inadecuado y se ayudara a los estudiantes con ideas afines a ser nombrados profesores y catedráticos en las universidades.

Nohl editó la serie de libros y el Manual y Flitner, su antiguo alumno, llegó a ser editor jefe del periódico. En un artículo principal publicado en 1928, Flitner $^{83}$ afirmaba que la educación nunca debía tomar parte en ninguna disputa. La educación debía estar orientada hacia un mundo superior de plenitud, el del auténtico pueblo: «Por encima de las discusiones terrenales, el educador tiene un modelo superior para orientarlo dentro de toda esta confusión: la auténtica comunidad ${ }^{84}$. Poco después, en 1933 , los nacionalsocialistas llegaron al poder. El análisis de Ringer ${ }^{85}$ de que este año de 1933 marcó el final de los intelectuales

${ }_{81}$ NoHL: 1926, p. 27, traducción libre.

82 Nohl, H.: «Die Theorie der Bildung», en Nohl, H. y Pallat, L. (eds.): Handbuch der Pädagogik, Band I: Die Theorie und die Entwicklung des Bildungswesens, Langensalza, Julius Beltz, 1933, pp. 3-80.

${ }_{83}$ Flitner, W.: «Zum Begriff der pädagogischen Autonomie», en Hermann, U. (ed.): Wilhelm Flitner: Gesammelte Schriften. Band 3: TheoretischeSchriften, Paderborn, Schöningh, 1989, pp. 237252 (Trabajo originalmente publicado en 1928 ).

${ }^{8}$ Ibidem, p. 244.

85 RINGER, Fritz: The decline of the German mandarins: The German academic community, I89o1933, Cambridge, MA, Harvard University Press, 1969 (El ocaso de los mandarines alemanes: catedráticos, profesores y la comunidad académica alemana, I890-1933, Barcelona, Pomares-Corredor, 1995). 


\section{LOS FUNDAMENTOS RELIGIOSOS DE LA BILDUNG COMO EPÍTOME DE LA EDUCACIÓN I42 ALEMANA Y SU FUNDAMENTAL RECELO ANTIOCCIDENTAL \\ DANIEL TRÖHLER}

alemanes es cierto -con la excepción de la gesitedwissenschaftliche Pädagogik-. A diferencia de muchos intelectuales de la época, Flitner nunca fue expulsado de su cátedra de la Universidad de Hamburgo; se retiró en 1958. Su profesor, Nohl, enseñó hasta 1937, fue reactivado en 1945 y se retiró en 1947. Spranger estaba en Japón como lector invitado en 1936/37 por orden del régimen nazi, y en 1943 editó los Discursos a la nación alemana de Fichte de nuevo con una introducción ${ }^{86}$. En 1945 fue nombrado presidente provisional de la Universidad de Berlín y en 1946 catedrático de Filosofía, Educación y Psicología en la Universidad de Tubinga; se retiró en 1950. Desde Tubinga, Spranger influyó en el nombramiento de profesores durante el período de posguerra con la ayuda de sus viejos compañeros ${ }^{87} \mathrm{y}$ aseguró la sostenibilidad del geisteswissenschaftliche Pädagogik. El periódico La Educación - Periódico mensual para la interrelación entre Cultura y Educación en Ciencia y la Vida (Die Erziebung - Monatsschrift für den Zusammenhang von Kultur und Erziehung in Wissenschaft und Leben) había finalizado en 1943, pero en 1945 Hohl fundó la revista Die Sammlung ( La colección) junto con sus antiguos estudiantes Flitner, Weniger y Otto Friedrich Bollnow. Nohl escribió el prefacio, que recuerda a sus escritos de i9I8. La revista servía a la reconstrucción de nuestra gente, su cultura y su nueva educación. Su objetivo era ser moralidad sencilla y firme fe en la eternidad de nuestro mundo espiritual-intelectual. El propósito era liderar los pensamientos de la gente hacia la esfera interior y ayudar a los profesores en su misión, que es incomparable a cualquier otra misión anterior ${ }^{88}$.

Paralelamente a Die Sammlung, la joven generación fundó Zeitschrift für Pädagogik (Revista de Pedagogía) en 1955: incluía a los discípulos de Nohl Flitner, Weniger, Bollnow y Fritz Blättner, que habían completado su habilitación con Flitner en Hamburgo en 1936 y Josef Dolch, que era el único católico en este ilustre círculo de protestantes luteranos. Apuntaban a una mayor calidad científica del discurso educativo y aceptaban con ese propósito incluso la investigación empírica, tal y como Flitner indicaba en la introducción, siempre que estuviera sostenida por reflexiones filosóficas. Sin embargo, los académicos más importantes siguieron siendo "Spranger, Nohl, Litt ${ }^{89}$ y no se detectó ni un solo atisbo de discusión extranjera ${ }^{9}$.

Dominaba esta generación cuando en los años sesenta -finalmente- la formación de los maestros de escuela elemental se localizó en las universidades. Se dio acceso a la docencia universitaria a profesores de los colegios de profesores,

86 Fichte, Johann Gottlieb: Reden an die deutsche Nation. Mit einem Geleitwort von Eduard Spranger, Leipzig, Meiner, 1943 (Trabajo publicado originalmente en I808).

${ }_{87}$ Kersting, Christa: Pädagogik im Nachkriegsdeutschland: Wissenschaftspolitik und Disziplinentwicklung 1945 bis 1955, Bad Heilbrunn, Klinkhardt, 2008.

88 Nohl, Herman: [Geleitwort zur neuen Zeitschrift Die Sammlung], Die Sammlung, I (1945), s. p.

89 Flitner, Wilheim: «Zur Einführung», Zeitschrift für Pädagogik, I (1955), pp. I-4, 4.

90 Andreas, hijo de Wilhelm Flitner (1922), llegó a ser sucesor de Spranger como profesor de Pedagogía en la Universidad de Tubinga entre 1958-1990. En I970 y 1975, uno de sus ayudantes era el anteriormente mencionado Ulrich Hermann (1939), quien completó su habilitación bajo la supervisión de Flitner. 
constituyendo una nueva generación de profesores universitarios en Pedagogía o Ciencias de la Educación, cuyos miembros más jóvenes se habían retirado a lo largo de los diez últimos años. Son ellos, nacidos al final y poco después de la Segunda Guerra Mundial, y sus discípulos quienes aún identifican los elementos centrales de la teoría de la Bildung y del ideal de Persönlichkeit usando esta batalla como reclamo contra PISA y otros fenómenos comparables. Si han sido capaces de historiar su propia socialización académica y cultural, pueden acaso haber sido capaces de encontrar muchos mejores argumentos contra PISA que los de Bildung y Persönlichkeit, ya que el informe PISA es comparable a una misión protestante, pero calvinista y no luterana ${ }^{91}$. El futuro mostrará hasta qué punto la nueva generación de académicos alemanes se ven estimulados por la investigación internacional e historian su propia tradición, abriendo una tercera vía -más allá de los dos grandes legados protestantes-internacional, comparativa e histórica; el único camino si la Pedagogía o las Ciencias de la Educación pretenden ser una disciplina académica que se tome en serio en las universidades. 